\title{
QUASICONFORMAL MAPPINGS WITH CONTROLLED LAPLACIAN AND HÖLDER CONTINUITY
}

\author{
David Kalaj and Arsen Zlatičanin \\ School of Mathematical Sciences, Huaqiao University, Quanzhou 362021, P. R. China \\ Permanent position: University of Montenegro, Faculty of Natural Sciences and Mathematics \\ Dzordza Vasingtona b.b. 81000 Podgorica, Montenegro; davidk@ucg.ac.me \\ University Luigj Gurakuqi, Department of Mathematics \\ Shkodra, Albania; arsen_zn@yahoo.fr
}

\begin{abstract}
We prove that every $K$-quasiconformal mapping $w$ of the unit ball $\mathbf{B} \subset \mathbf{R}^{n}, n \geq 2$ onto a $C^{2}$-Jordan domain $\Omega$ is Hölder continuous with constant $\alpha=2-\frac{n}{p}$, provided its weak Laplacian $\Delta w$ is in $L^{p}\left(\mathbf{B}^{n}\right)$ for some $n / 2<p<n$. In particular it is Hölder continuous for every $0<\alpha<1$ provided that $\Delta w \in L^{n}\left(\mathbf{B}^{n}\right)$. Finally for $p>n$, we prove that $w$ is Lipschitz continuous, a result, whose proof has been already sketched in [16] by the first author and Saksman. The paper contains the proofs of some results announced in [17].
\end{abstract}

\section{Introduction}

In the paper $\mathbf{B}^{n}$ is the unit ball in $\mathbf{R}^{n}, n \geq 2$ and $\mathbf{S}^{n-1}$ is the unit sphere. We consider the vector norm $|x|=\left(\sum_{i=1}^{n} x_{i}^{2}\right)^{1 / 2}$ and the matrix norms $|A|=\sup \{|A x|:|x|=$ 1\}. A homeomorphism $u: \Omega \rightarrow \Omega^{\prime}$ between two open subsets $\Omega$ and $\Omega^{\prime}$ of Euclidean space $\mathbf{R}^{n}$ will be called a $K(K \geq 1)$ quasiconformal or shortly a qc mapping if the following two conditions are satisfied:

(i) $u$ is absolutely continuous function in almost every segment parallel to some of the coordinate axes and there exist the partial derivatives which are locally $L^{n}$ integrable functions on $\Omega$. We will write $u \in A C L^{n}$.

(ii) $u$ satisfies the condition

$$
|\nabla u(x)|^{n} / K \leq J_{u}(x) \leq K l(\nabla u(x))^{n},
$$

at almost every $x$ in $\Omega$ where

$$
l(\nabla u(x)):=\inf \{|\nabla u(x) \zeta|:|\zeta|=1\}
$$

and $J_{u}(x)$ is the Jacobian determinant of $u$ (see [26]).

Notice that, for a continuous mapping $u$ the condition (i) is equivalent to the condition that $u$ belongs to the Sobolev space $W_{\text {loc }}^{1, n}(\Omega)$.

Let $P$ be Poisson kernel i.e. the function

$$
P(x, \eta)=\frac{1-|x|^{2}}{|x-\eta|^{n}},
$$

and let $G$ be the Green function i.e. the function

$$
G(x, y)=c_{n} \begin{cases}\left(\frac{1}{|x-y|^{n-2}}-\frac{1}{(|x| y|-y /| y||)^{n-2}}\right), & \text { if } n \geq 3 \\ \log \frac{|x-y|}{|1-x \bar{y}|}, & \text { if } n=2 \text { and } x, y \in \mathbf{C} \cong \mathbf{R}^{2}\end{cases}
$$

https://doi.org/10.5186/aasfm.2019.4440

2010 Mathematics Subject Classification: Primary 30C65.

Key words: Lipschitz continuity, Poisson equation. 
where $c_{n}=\frac{1}{(n-2) \Omega_{n-1}}$, and $\Omega_{n-1}$ is the measure of $\mathbf{S}^{n-1}$. Both $P$ and $G$ are harmonic for $|x|<1, x \neq y$.

Let $p>1$ and $f \in L^{p}\left(\mathbf{S}^{n-1}, \mathbf{R}^{n}\right)$. Further let $g: \mathbf{B}^{n} \mapsto \mathbf{R}^{n}$ be continuous. The weak solution of the equation (in the sense of distributions) $\Delta u=g$ in the unit ball satisfying the boundary condition $\left.u\right|_{\mathbf{S}^{n-1}}=f \in L^{1}\left(\mathbf{S}^{n-1}\right)$ is given by

$$
u(x)=P[f](x)-G[g](x):=\int_{\mathbf{S}^{n-1}} P(x, \eta) f(\eta) d \sigma(\eta)-\int_{\mathbf{B}^{n}} G(x, y) g(y) d y,
$$

$|x|<1$. Here $d \sigma$ is the $n-1$ dimensional Hausdorff measure of Euclidean sphere satisfying the condition: $P[1](x) \equiv 1$. It is well known that if $f$ and $g$ are continuous in $\mathbf{S}^{n-1}$ and in $\overline{\mathbf{B}^{n}}$ respectively, then the mapping $u=P[f]-G[g]$ has a continuous extension $\tilde{u}$ to the boundary and $\tilde{u}=f$ on $\mathbf{S}^{n-1}$. If $g \in L^{\infty}$ then $G[g] \in C^{1, \alpha}\left(\overline{\mathbf{B}^{n}}\right)$. See [6, Theorem 8.33] for this argument.

We will consider the solutions of the PDE $\Delta u=g$ that are quasiconformal as well and investigate their Lipschitz and Hölder character. A mapping $f$ of a set $\Omega$ in Euclidean $n$-space $\mathbf{R}^{n}$ into $\mathbf{R}^{n}, n \geq 2$, is said to belong to the Hölder class $\operatorname{Lip}_{\alpha}(\Omega)$, $0<\alpha \leq 1$, if there exists a constant $M>0$ such that

$$
|f(x)-f(y)| \leq M|x-y|^{\alpha}
$$

for $x, y \in \Omega$. If $D$ is a bounded domain in $\mathbf{R}^{n}$ and if $f$ is quasiconformal in $D$ with $f(D) \subset \mathbf{R}^{n}$, then $f$ is in $\operatorname{Lip}_{\alpha}(A)$ for each compact $A \subset D$, where $\alpha=K_{I}(f)^{1 /(1-n)}$ and $K_{I}(f)$ is the inner dilatation of $f$. Simple examples show that $f$ need not be in $\operatorname{Lip}_{\alpha}(D)$ even when $f$ is continuous in $\bar{D}$.

However, Martio and Näkki in [20] showed that if $f$ induces a boundary mapping which belongs to $\operatorname{Lip}_{\alpha}(\partial D)$, then $f$ is in $\operatorname{Lip}_{\beta}(D)$, where

$$
\beta=\min \left(\alpha, K_{I}(f)^{1 /(1-n)}\right) ;
$$

the exponent $\beta$ is sharp. In a recent paper of the first author and Saksman [16] it is proved the following result, if $f$ is quasiconformal mapping of the unit disk $\mathbf{B}^{2}$ onto a Jordan domain with $C^{2}$ boundary such that its weak Laplacian $\Delta f \in L^{p}\left(\mathbf{B}^{2}\right)$, for $p>2$, then $f$ is Lipschitz continuous. The condition $p>2$ is necessary also. Further in the same paper they proved that if $p=1$, then $f$ is absolutely continuous on the boundary of $\partial \mathbf{B}^{2}$.

The results from [16] generalize in certain sense the results of the first author, Mateljević, Božin, Pavlović, Partyka, Sakan, Manojlović, Astala [10, 11, 14, 13, 22, $21,23,24,8,9,3]$, since they do not assume that the mapping is harmonic, neither its weak Laplacian is bounded. The topic has its origin in the classical paper by Martio [18].

In this paper we consider the following problem (a question). To what extent the condition $1<p<2$ affects to the boundary behaviour of quasiconformal mappings between Euclidean domains with smooth boundaries?

We are interested in the condition under which the quasiconformal mapping is in $\operatorname{Lip}_{\alpha}\left(\mathbf{B}^{n}\right)$, for every $\alpha<1$. It follows form our results that if $u$ is a quasiconformal selfmapping of the unit ball and $|\Delta u| \in L^{p}$ with $p>n / 2$ then $u$ is in $\operatorname{Lip}_{\alpha}\left(\mathbf{B}^{n}\right)$, where $\alpha=2-\frac{p}{n}$. In particular if $p=n$, then $u \in \operatorname{Lip}_{\alpha}\left(\mathbf{B}^{n}\right)$ for $\alpha<1$.

Our result is the following:

Theorem 1. Let $n \geq 2$ and let $p>n / 2$ and assume that $g \in L^{p}\left(\mathbf{B}^{n}\right)$. Assume that $w$ is a $K$-quasiconformal solution of $\Delta w=g$, that maps the unit ball onto a bounded Jordan domain $\Omega \subset \mathbf{R}^{n}$ with $C^{2}$-boundary. 
(1) If $p<n$, then $w$ is Hölder continuous with the Hölder constant $\alpha=2-\frac{n}{p}$.

(2) If $p=n$, then $w$ is Hölder continuous for every $\alpha \in(0,1)$.

(3) If $p>n$, then $w$ is Lipschitz continuous.

Remark 1.1. The item (3) of the previous theorem for $n=2$ has been already proved in [16], where it is also mentioned that a similar approach can be used for $n>2$. The main novelty of this paper are items (1) and (2). The proof presented in this paper uses some iterating schema that already appeared in the papers by Astala and Manojlović [3] and by the first author and Saksman in [16]. Astala and Manojlović in [3] among the other results presented a short proof of the fact that every harmonic mapping of the unit ball onto a Jordan domain with $C^{2}$ is Lipschitz continuous. This result has been firstly proved by Kalaj in [10].

The formulations of results of this paper and the formulations of some necessary lemmas has been published in the conference paper [17], but without proofs. The detailed proofs are given here. Motivated by the corresponding result for the planar case in [16], we conjecture that

Conjecture 1.1. Under condition of Theorem 1 , for $p=n / 2, n \geq 3$, the mapping $w$ is absolutely continuous on every line from the boundary of the unit ball.

\section{Proofs of the results}

In what follows, we say that a bounded Jordan domain $\Omega \subset \mathbf{R}^{n}$ has $C^{2}$-boundary if it is the image of the unit ball $\mathbf{B}^{n}$ under a $C^{2}$-diffeomorphism of the whole Euclidean space onto itself. In the sequel, $\Delta$ refers to the distributional Laplacian. We shall make use of the following well-known facts from potential theory:

Proposition 2.1. (Morrey's inequality) Assume that $n<p \leq \infty$ and assume that $U$ is a domain in $\mathbf{R}^{n}$ with $C^{1}$ boundary. Then there exists a constant $C$ depending only on $n, p$ and $U$ so that

$$
\|u\|_{C^{0, \alpha}(U)} \leq C\|u\|_{W^{1, p}(U)}
$$

for every $u \in C^{1}(U) \cap L^{p}(U)$, where

$$
\alpha=1-\frac{n}{p} .
$$

Lemma 1. (See e.g. [3]) Suppose that $\omega \in W_{\text {loc }}^{2,1}\left(\mathbf{B}^{n}\right) \cap C\left(\overline{\mathbf{B}^{n}}\right)$, that $h \in L^{p}\left(\mathbf{B}^{n}\right)$ for some $1<p<\infty$ and that

$$
\Delta \omega=h \text { in } \mathbf{B}^{n}, \quad \text { with }\left.\omega\right|_{\mathbf{S}^{n-1}}=0 .
$$

a) If $1<p<n$, then

$$
\|\nabla \omega\|_{L^{q}\left(\mathbf{B}^{n}\right)} \leq c(p, n)\|h\|_{L^{p}\left(\mathbf{B}^{n}\right)}, \quad q=\frac{p n}{n-p} .
$$

b) If $p=n$ and $1<q<\infty$, then

$$
\|\nabla \omega\|_{L^{q}\left(\mathbf{B}^{n}\right)} \leq c(q, n)\|h\|_{L^{n}\left(\mathbf{B}^{n}\right)} .
$$

c) if $p>n$, then

$$
\|\nabla \omega\|_{L^{\infty}\left(\mathbf{B}^{n}\right)} \leq c(p, n)\|h\|_{L^{n}\left(\mathbf{B}^{n}\right)} .
$$

Now we prove

Lemma 2. If $\Delta u=g \in L^{p}$ and $r<1$, then $D u \in L^{q}(r \mathbf{B})$ for $q \leq \frac{n p}{n-p}$. 
Proof of Lemma 2. By writing $u=v+w$ from (2), and differentiating it we have

$$
D u(x)=D v+D w=\int_{\mathbf{S}^{n-1}} \nabla_{x} P(x, \eta) f(\eta) d \sigma(\eta)-\int_{\mathbf{B}} \nabla_{x} G(x, y) g(y) d y .
$$

Then

$$
\int_{r \mathbf{B}}|D u(x)|^{q} d x=\int_{r \mathbf{B}}\left|\int_{\mathbf{S}^{n-1}} \nabla_{x} P(x, \eta) f(\eta) d \sigma(\eta)-\int_{\mathbf{B}} \nabla_{x} G(x, y) g(y) d y\right|^{q} d x .
$$

Thus

$$
\begin{aligned}
& \|D u\|_{L^{q}(r \mathbf{B})}=\|D v\|_{L^{q}(r \mathbf{B})}+\|D w\|_{L^{q}(r \mathbf{B})} \\
& \leq\left(\int_{r \mathbf{B}}\left|\int_{\mathbf{S}^{n-1}} \nabla_{x} P(x, \eta) f(\eta) d \sigma(\eta)\right|^{1 / q}\right)^{1 / q}+\left(\int_{r \mathbf{B}}\left|\int_{\mathbf{B}} \nabla_{x} G(x, y) g(y) d y\right|^{q} d x\right)^{1 / q} .
\end{aligned}
$$

Further, there is a constant $C$ so that

$$
\left|\nabla_{x} P(x, \eta)\right| \leq \frac{C}{(1-|x|)^{n+1}} .
$$

From Lemma 1 and (6) we have $\|D u\|_{L^{q}(r \mathbf{B})}<\infty$.

Now we formulate the following fundamental result of Gehring:

Proposition 2.2. [5] Let $f$ be a quasiconformal mapping of the unit ball $\mathbf{B}^{n}$ onto a Jordan domain $\Omega$ with $C^{2}$ boundary. Then there is a constant $p=p(K, n)>n$ so that

$$
\int_{\mathbf{B}^{n}}|D f|^{p}<C(n, K, f(0), \Omega)
$$

Then we prove

Lemma 3. If $H: \mathbf{R}^{n} \rightarrow \mathbf{R}$ and $w=\left(w_{1}, \ldots, w_{n}\right): A \rightarrow B$ (where $A, B$ are open subsets in $\mathbf{R}^{n}$ ) are functions $C^{2}$ mappings, then

$$
\Delta(H \circ w)=\sum_{i=1}^{n} \frac{\partial^{2} H}{\partial w_{i}^{2}}\left|\nabla w_{i}\right|^{2}+2 \sum_{1 \leq i<j \leq n} \frac{\partial^{2} H}{\partial w_{i} \partial w_{j}}\left\langle\nabla w_{i}, \nabla w_{j}\right\rangle+\sum_{i=1}^{n} \frac{\partial H}{\partial w_{i}} \Delta w_{i}
$$

Proof. For every $k \in(1, \ldots, n)$ we have:

$$
\frac{\partial(H \circ w)\left(x_{1}, \ldots, x_{n}\right)}{\partial x_{k}}=\sum_{i=1}^{n} \frac{\partial H}{\partial w_{i}} \frac{\partial w_{i}}{\partial x_{k}} .
$$

Thus

$$
\begin{aligned}
\frac{\partial^{2}(H \circ w)\left(x_{1}, \ldots, x_{n}\right)}{\partial x_{k}^{2}} & =\sum_{i=1}^{n} \frac{\partial\left[\frac{\partial H}{\partial w_{i}} \frac{\partial w_{i}}{\partial x_{k}}\right]}{\partial x_{k}} \\
& =\sum_{i=1}^{n}\left[\frac{\partial\left[\frac{\partial H}{\partial w_{i}}\right]}{\partial x_{k}} \frac{\partial w_{i}}{\partial x_{k}}+\frac{\partial H}{\partial w_{i}} \frac{\partial^{2} w_{i}}{\partial x_{k}^{2}}\right] \\
& =\sum_{i=1}^{n}\left[\left[\sum_{j=1}^{n} \frac{\partial^{2} H}{\partial w_{j} \partial w_{i}} \frac{\partial w_{j}}{\partial x_{k}}\right] \frac{\partial w_{i}}{\partial x_{k}}\right]+\sum_{i=1}^{n} \frac{\partial H}{\partial w_{i}} \frac{\partial^{2} w_{i}}{\partial x_{k}^{2}} \\
& =\sum_{i, j=1}^{n} \frac{\partial^{2} H}{\partial w_{i} \partial w_{j}}\left[\frac{\partial w_{i}}{\partial x_{k}} \frac{\partial w_{j}}{\partial x_{k}}\right]+\sum_{i=1}^{n} \frac{\partial H}{\partial w_{i}} \frac{\partial^{2} w_{i}}{\partial x_{k}^{2}}
\end{aligned}
$$


Now we have:

$$
\begin{aligned}
\Delta(H \circ w) & =\sum_{k=1}^{n} \frac{\partial^{2}(H \circ w)\left(x_{1}, \ldots, x_{n}\right)}{\partial x_{k}^{2}} \\
& =\sum_{k=1}^{n}\left[\sum_{i, j=1}^{n} \frac{\partial^{2} H}{\partial w_{i} \partial w_{j}}\left[\frac{\partial w_{i}}{\partial x_{k}} \frac{\partial w_{j}}{\partial x_{k}}\right]+\sum_{i=1}^{n} \frac{\partial H}{\partial w_{i}} \frac{\partial^{2} w_{i}}{\partial x_{k}^{2}}\right] \\
& =\sum_{i, j=1}^{n} \frac{\partial^{2} H}{\partial w_{i} \partial w_{j}}\left[\sum_{k=1}^{n} \frac{\partial w_{i}}{\partial x_{k}} \frac{\partial w_{j}}{\partial x_{k}}\right]+\sum_{i=1}^{n} \frac{\partial H}{\partial w_{i}}\left[\sum_{k=1}^{n} \frac{\partial^{2} w_{i}}{\partial x_{k}^{2}}\right] \\
& =\sum_{i=1}^{n} \frac{\partial^{2} H}{\partial w_{i}^{2}}\left|\nabla w_{i}\right|^{2}+2 \sum_{1 \leq i<j \leq n} \frac{\partial^{2} H}{\partial w_{i} \partial w_{j}}\left\langle\nabla w_{i}, \nabla w_{j}\right\rangle+\sum_{i=1}^{n} \frac{\partial H}{\partial w_{i}} \Delta w_{i} .
\end{aligned}
$$

Proof of Theorem 1. It turns out that the approach of [8], where the use of distance functions was initiated, is substantial for further development.

We say $a \approx b$ if there is a constant $C \geq 1$ such that $a / C \leq b \leq C a$; and we say $a \lesssim b$ if there is a constant $C>0$ such that $a \leq C b$.

By our assumption on the domain, we may fix a diffeomorphism $\psi: \bar{\Omega} \rightarrow \overline{\mathbf{B}^{n}}$ that is $C^{2}$ up to the boundary. Denote $H:=1-|\psi|^{2}$, whence $H$ is $C^{2}$-smooth in $\bar{\Omega}$ and vanishes on $\partial \Omega$ with $|\nabla H| \approx 1$ in a neighborhood of $\partial \Omega$. We may then define $h: \mathbf{B}^{n} \rightarrow[0,1]$ by setting

$$
h(z):=H \circ w(z)=1-|\psi(w(z))|^{2} \text { for } z \in \mathbf{B}^{n} .
$$

The quasiconformality of $w$ and the behavior of $\nabla H$ near $\partial \Omega$ imply that there is $r_{0} \in(0,1)$ so that the weak gradients satisfy

$$
|\nabla h(x)| \approx|\nabla w(x)| \text { for } r_{0} \leq|x|<1 .
$$

Moreover, by Lemma 2, for $q \in\left(1, \frac{n p}{n-p}\right]$, we have

$$
\|\nabla h(x)\|_{L^{q}\left(r_{0} \mathbf{B}^{n}\right)} \lesssim\|\nabla w(x)\|_{L^{q}\left(r_{0} \mathbf{B}^{n}\right)} \leq C .
$$

It follows that for any $q \in\left(1, \frac{n p}{n-p}\right]$ we have that

$$
\nabla h \in L^{q}\left(\mathbf{B}^{n}\right) \quad \text { if and only if } \nabla w \in L^{q}\left(\mathbf{B}^{n}\right) .
$$

From Lemma 3 by using the fact that $H \in C^{2}$ is a real valued function, we obtain

$$
|\Delta h| \lesssim|\nabla w|^{2}+|g|
$$

The higher integrability of quasiconformal self-maps of $\mathbf{B}^{n}$ makes sure that $\nabla(\psi \circ$ $w) \in L^{q}\left(\mathbf{B}^{n}\right)$ for some $q>n$, which implies that $\nabla w \in L^{q}\left(\mathbf{B}^{n}\right)$. By combining this with the fact that $g \in L^{p}\left(\mathbf{B}^{n}\right)$ with $p>n / 2$, we deduce that $\Delta h \in L^{r}\left(\mathbf{B}^{n}\right)$ with $r=\min (p, q / 2)>n / 2$. As in [3] and [16], we use bootstrapping argument which can be stated as the following observation.

$$
\text { If } \nabla w \in L^{q}\left(\mathbf{B}^{n}\right) \text { with } n<q<2 n \text {, then } \nabla w \in L^{\frac{n a}{2 n-a}}\left(\mathbf{B}^{n}\right) \text {, }
$$

where $a=q \wedge 2 p:=\min \{q, 2 p\}$. In order to prove (10), assume that $\nabla w \in L^{q}\left(\mathbf{B}^{n}\right)$ for an exponent $q \in(n, 2 n)$. Then (9) and our assumption on $g$ verify that $\Delta h \in$ $L^{q / 2 \wedge p}\left(\mathbf{B}^{n}\right)$. Since $h$ vanishes continuously on the boundary $\partial \mathbf{B}^{n}$, we may apply Lemma 1 (a) to obtain that $\nabla h \in L^{n a /(2 n-a)}\left(\mathbf{B}^{n}\right)$ which yields the claim according to $(8)$. 
We then claim that in our situation one has $\nabla w \in L^{q}\left(\mathbf{B}^{n}\right)$ with some exponent

$$
q \begin{cases}=2 p, & \text { if } p \leq n \\ >2 n, & \text { if } p>n .\end{cases}
$$

To prove that, fix an exponent $q_{0}>n$ obtained from the higher integrability of the quasiconformal map $w$ so that $\nabla w \in L^{q_{0}}\left(\mathbf{B}^{n}\right)$. By diminishing $q_{0}$ if needed, we may assume that $q_{0} \in(n, 2 n)$ and $q_{0} \notin\left\{2^{m} /\left(2^{m-1}-1\right), m=3,4, \ldots\right\}$. Then we may iterate (10) and deduce inductively that $\nabla w \in L^{a_{k}}\left(\mathbf{B}^{n}\right)$ for $k=0,1,2, \ldots, k_{0}$, where $a_{0}=q_{0} \wedge 2 p$ and $a_{k}$ satisfy the recursion $a_{k+1}=\frac{n a_{k}}{2 n-a_{k}} \wedge 2 p$ and $k_{0}$ is the first index such that

$$
a_{k_{0}} \begin{cases}=2 p, & \text { if } p \leq n \\ >2 n, & \text { if } p>n .\end{cases}
$$

Such an index exists. Namely if $p>n$ and $a_{k} \leq 2 n$, then $a_{k}<a_{k+1}=\frac{n a_{k}}{2 n-a_{k}}$. If for all $k, a_{k} \leq 2 n$, then $a_{k}$ converges to a point $a$ so that $\frac{n a}{2 n-a}=a$, which is then equal to $n$ and this is impossible. If $p \leq n$, then again $a_{k}$ is nondecreasing, which means that it converges to $a=n$, which is impossible, or which is stationary sequence from an index $k_{0}$, and then $a_{k}=2 p$ for $k \geq k_{0}$.

Thus we may assume that $\nabla w \in L^{q}\left(\mathbf{B}^{n}\right)$, where $q=a_{k_{0}}$ satisfies (11). Now we consider the following cases.

The case $p<n$. Since $\nabla w \in L^{n p /(n-p)}\left(\mathbf{B}^{n}\right)$, by Morrey's inequality $w$ is Hölder continuous with the constant $c=\alpha=2-\frac{n}{p}$ as claimed.

The case $p=n$, in this case we use the previous case by choosing $p^{\prime}<p$ close enough to $p$.

The case $p>n$. We know that $\nabla w \in L^{q}$ with some $q>2 n$. Further (9) shows that $\Delta h \in L^{p \wedge(q / 2)}\left(\mathbf{B}^{n}\right)$. As $p \wedge(q / 2)>2 n$, Lemma $1(\mathrm{c})$ verifies that $\nabla h \in L^{\infty}\left(\mathbf{B}^{n}\right)$. Thus $\nabla w \in L^{\infty}\left(\mathbf{B}^{n}\right)$ and hence $w$ is Lipschitz continuous.

If follows from the proof of the previous theorem the following theorem.

Theorem 2. Assume that $g \in L^{n}\left(\mathbf{B}^{n}\right)$. If $w$ is a $K$-quasiconformal solution of $\Delta w=g$, that maps the unit disk onto a bounded Jordan domain $\Omega \subset \mathbf{R}^{n}$ with $C^{2}$-boundary, then $D w \in L^{p}\left(\mathbf{B}^{n}\right)$ for every $p<\infty$.

\section{References}

[1] Agmon, S., A. Douglis, and L. Nirenberg: Estimates near the boundary for elliptic partial differential equations satisfying general bounday conditions. I. - Comm. Pure Appl. Math. 12, 1959, 623-727.

[2] Ahlfors, L: Lectures on quasiconformal mappings. - Van Nostrand Mathematical Studies, D. Van Nostrand, 1966.

[3] Astala, K., and V. Manojlović: On Pavlović theorem in space. - Potential Anal. 43:3, $2015,361-370$.

[4] BožIn, V., and M. Mateljević: Quasiconformal and HQC mappings between Lyapunov Jordan domains. - arXiv:1805.04313.

[5] Gehring, F. W.: The $L^{p}$-integrability of the partial derivatives of a quasiconformal mapping. - Acta Math. 130, 1973, 265-277.

[6] Gilbarg, D., and N. Trudinger: Elliptic partial differential equations of second order. 2nd Edition. - Springer, 1977, 1983. 
[7] Kahane, J. P.: Trois notes sur les ensembles parfait linearés. - Enseign. Math. 15, 1969, $185-192$.

[8] Kalaj, D.: Harmonic mappings and distance function. - Ann. Sc. Norm. Super. Pisa Cl. Sci. (5) 10, 2011, 669-681.

[9] KalaJ, D.: On boundary correspondences under quasiconformal harmonic mappings between smooth Jordan domains. - Math. Nachr. 285:2-3, 2012, 283-294.

[10] KalaJ, D.: A priori estimate of gradient of a solution to certain differential inequality and quasiregular mappings. - J. Anal. Math. 119, 2013, 63-88.

[11] KalaJ, D.: Quasiconformal harmonic mappings between Dini's smooth Jordan domains. Pacific J. Math. 276:1, 2015, 213-228.

[12] Kalaj, D., M. Markovic, and M. Mateljević: Carathéodory and Smirnov type theorems for harmonic mappings of the unit disk onto surfaces. - Ann. Acad. Sci. Fenn. Math. 38, 2013, $565-580$.

[13] Kalaj, D., and M. MatelJević: Inner estimate and quasiconformal harmonic maps between smooth domains. - J. Anal. Math. 100, 2006.

[14] Kalaj, D., and M. Mateljević: $\left(K, K^{\prime}\right)$-quasiconformal harmonic mappings. - Potential Anal. 36:1, 2012, 117-135.

[15] Kalaj, D., and M. Pavlović: On quasiconformal self-mappings of the unit disk satisfying the Poisson equation. - Trans. Amer. Math. Soc. 363, 2011, 4043-4061.

[16] Kalaj, D., and E. Saksman: Quasiconformal mappings with controlled Laplacian. - J. Anal. Math. (to appear).

[17] Kalaj, D., and A. Zlatičanin: Hölder continuity of quasiconformal mappings. - In: Proceedings of the OPTIMA 2017 conference (Petrovac, Montenegro), edited by Yu. G. Evtushenko, M. Yu. Khachay, O. V. Khamisov, Yu. A. Kochetov, V. U. Malkova, and M. A. Posypkin, 2017.

[18] Martio, O.: On harmonic quasiconformal mappings. - Ann. Acad. Sci. Fenn. Ser. A I Math. $425,1968,3-10$.

[19] Martio, O., and R. NÄKKI: Continuation of quasiconformal mappings. - Sibirsk. Mat. Zh. 28:4, 1987, 162-170 (in Russian).

[20] Martio, O., and R. NÄKKI: Boundary Hölder continuity and quasiconformal mappings. - J. London Math. Soc. (2) 44:2, 1991, 339-350.

[21] Partyka, D., and K. SAKAn: On bi-Lipschitz type inequalities for quasiconformal harmonic mappings. - Ann. Acad. Sci. Fenn. Math. 32, 2007, 579-594.

[22] Partyka, D., and K. SAKan: Heinz type inequalities for Poisson integrals. - Comput. Methods Funct. Theory 14:2-3, 2014, 219-236.

[23] Partyka, D., and K. SAKan: Quasiconformal and Lipschitz harmonic mappings of the unit disk onto bounded convex domains. - Ann. Acad. Sci. Fenn. Math. 39:2, 2014, 811-830.

[24] PAVlović, M.: Boundary correspondence under harmonic quasiconformal homeomorfisms of the unit disc. - Ann. Acad. Sci. Fenn. Math. 27, 2002, 365-372.

[25] Pommerenke, Ch.: Boundary behaviour of conformal maps. - Grundlehren Math. Wiss. 299, Berlin, Springer-Verlag, 1992.

[26] Reshetnyak, Yu. G.: Generalized derivatives and differentiability almost everywhere. - Mat. Sb. (N.S.) 75:117, 1968, 323-334 (in Russian).

Received 16 July 2018 • First revised received 9 November 2018

Second revised received 29 November 2018 • Accepted 10 December 2018 